\title{
Eficácia do tratamento do tabagismo na perspectiva da redução de danos e do cuidado farmacêutico
}

\author{
Effectiveness of smoking treatment from the perspective of harm reduction and \\ pharmaceutical care
}
Efectividad del tratamiento para fumar desde la perspectiva de la reducción de daños y la atención farmacéutica

Maicon Brustolin ${ }^{1 *}$, Fernanda Almeida Fettermann², Raqueli Altamiranda Bittencourt ${ }^{3}$, Vanessa Bley Ribeiro'.

\section{RESUMO}

Objetivo: Avaliar a efetividade da assistência em saúde em controlar o tabagismo e analisar a influência de sintomas psicológicos associados a esse processo. Métodos: Assistência multiprofissional em saúde, por meio da redução de danos como os atendimentos individuais, terapia de grupo e disponibilização de medicamentos. Realizaram-se acompanhamento a 30 tabagistas, durante 60 dias, e analisou-se a efetividade da proposta através do teste de Fagerström. A influência de sintomas psicológicos foi investigada através do questionário EADS-21. Resultados: Os usuários apresentaram uma dependência elevada ou muito elevada no início do tratamento (73,3\%). A assistência prestada demonstrou-se efetiva, pois $50 \%$ dos pacientes interromperam o hábito de fumar. A farmacoterapia foi considerada a medida terapêutica mais efetiva pelos usuários, $o$ que ressalta a importância da assistência farmacêutica e do acompanhamento farmacoterapêutico. Observou-se que o controle do tabagismo resultou em uma melhora da saúde psicológica dos usuários, havendo uma diminuição dos sintomas de ansiedade, depressão e estresse. Conclusão: A assistência ofertada aos tabagistas foi efetiva, auxiliando de forma significativa o controle do tabagismo e contribuindo para uma diminuição dos riscos relacionados ao aparecimento de comorbidades físicas e mentais pelo uso do tabaco.

Palavras-Chave: Tabagismo, Tabaco, Dependência de Nicotina, Tratamento farmacológico, Assistência farmacêutica.

\begin{abstract}
Objective: To evaluate the effectiveness of health care in controlling smoking and to analyze the influence of psychological symptoms associated with this process. Methods: Multiprofessional health care, through harm reduction such as individual care, group therapy and medication availability. Thirty smokers were monitored for 60 days and the proposal's effectiveness was analyzed using the Fagerström test. The influence of psychological symptoms was investigated through the EADS-21 questionnaire. Results: Users had a high or very high dependence at the beginning of treatment (73.3\%). The assistance provided proved to be effective, as $50 \%$ of patients discontinued smoking. Pharmacotherapy was considered the most effective therapeutic measure by users, which underscores the importance of pharmaceutical care and pharmacotherapeutic monitoring. Smoking control resulted in an improvement in the psychological health of users, with a decrease in anxiety, depression and stress symptoms. Conclusion: The assistance offered to smokers was effective, significantly helping to control smoking and contributing to a decrease in risks related to the onset of physical and mental comorbidities due to tobacco use.
\end{abstract}

Key words: Smoking, Tobacco, Nicotine Dependence, Pharmacological Treatment, Pharmaceutical Care.

\footnotetext{
1 Universidade Federal do Pampa (UNIPAMPA), Uruguaiana- RS. *E-mail: maiconbrustolin@gmail.com

2 Universidade Federal do Rio Grande do Sul (UFRGS), Porto Alegre- RS.

${ }^{3}$ Prefeitura Municipal de Uruguaiana, Uruguaiana-RS
}

SUBMETIDO EM: 9/2019

ACEITO EM: 10/2019

PUBLICADO EM: 11/2019 


\section{RESUMEN}

Objetivo: Evaluar la efectividad de la atención de la salud en el control del tabaquismo y analizar la influencia de los síntomas psicológicos asociados con este proceso. Métodos: Atención de salud multiprofesional, a través de la reducción de daños, como atención individual, terapia grupal y disponibilidad de medicamentos. Treinta fumadores fueron monitoreados durante 60 días y la efectividad de la propuesta se analizó mediante la prueba de Fagerström. La influencia de los síntomas psicológicos se investigó a través del cuestionario EADS-21. Resultados: Los usuarios tenían una dependencia alta o muy alta al comienzo del tratamiento $(73,3 \%)$. La asistencia brindada demostró ser efectiva, ya que el $50 \%$ de los pacientes dejaron de fumar. La farmacoterapia fue considerada la medida terapéutica más efectiva por los usuarios, lo que subraya la importancia de la atención farmacéutica y el monitoreo farmacoterapéutico. El control del tabaquismo resultó en una mejora en la salud psicológica de los usuarios, con una disminución en los síntomas de ansiedad, depresión y estrés. Conclusión: La asistencia ofrecida a los fumadores fue efectiva, ayudando significativamente a controlar el tabaquismo y contribuyendo a una disminución de los riesgos relacionados con la aparición de comorbilidades físicas y mentales por el consumo de tabaco.

Palabras clave: Tabaquismo, tabaco, Dependencia de la nicotina, Tratamiento farmacológico, Atención farmacéutica.

\section{INTRODUÇÃO}

Na queima do tabaco são identificadas mais de quatro mil substâncias químicas, sendo que 43 dessas substâncias são cancerígenas. O componente mais destacável é a nicotina, um estimulante do sistema nervoso central que produz uma sensação prazerosa, de bem-estar e relaxamento em seus usuários, entretanto é também o principal agente relacionado ao desenvolvimento da dependência (ROSEMBERG J, 2004; OLIVEIRA F e COSTA MCF, 2012; PICCIOTTO MR e MINEU YS, 2014; MOREIRA EDT, 2007).

O consumo de tabaco é um importante fator de risco para o surgimento de aproximadamente 50 doenças, dentre as quais diversos tipos de cânceres, doenças respiratórias e cardiovasculares, que geram altos índices de mortalidade em razão da alta prevalência de fumantes (ANS, 2009). Estima-se que o tabaco é responsável pela morte de mais de sete milhões de pessoas por ano, sendo considerado a maior causa isolada evitável de mortes precoces em todo o mundo (INCA, 2017; WHO, 2017).

No Brasil, o percentual total de fumantes com 18 anos ou mais é de $10,4 \%$, sendo que homens consomem mais tabaco e derivados do que mulheres (VIGITEL, 2015). Ainda, o consumo do tabaco costuma ser mais incidente em indivíduos com sintomas psiquiátricos devido à percepção de que há um alívio do estresse e do nervosismo ao consumir o produto (BRASIL, 2001; RIAD-ALLEN L, 2017; PECKHAM E et al., 2015; BRESLAU N, NOVAK SP e KESSLER RC, 2004).

Atualmente o tabagismo é considerado uma doença crônica e integra o grupo de transtornos mentais e comportamentais devidos ao uso de substância psicoativa, portanto, uma dependência química, que envolve a inter-relação de fatores fisiológicos, psicológicos e comportamentais (BRASIL, 2001; WHO, 2003; AMERICAN PSYCHIATRIC ASSOCIATION, 2014). Conforme a Agência Nacional de Saúde Suplementar (ANS, 2009), a taxa de abstinência de quem tenta parar sozinho é significativamente menor (10,9\%) do que o tabagista que recebe um aconselhamento intensivo por mais de 10 minutos $(22,1 \%)$. Nesse contexto, ações direcionadas aos fumantes, no sentido de conscientizá-los sobre os riscos e malefícios ocasionados pelo uso do tabaco ao longo da vida, podem refletir em mudança de hábitos e, consequentemente, numa melhoria da qualidade de vida para estes usuários (WHO, 2003).

Diante disso, o presente estudo teve como objetivo avaliar a efetividade da assistência em saúde em controlar o tabagismo, bem como analisar a influência de sintomas psicológicos associados a esse processo.

\section{MÉTODOS}

Os tabagistas interessados em reduzir ou cessar o hábito de fumar foram convidados a participar do estudo no momento em que procuraram o Programa para Controle do Tabagismo de Uruguaiana (PCTU), nas 
dependências da Secretaria de Saúde de Uruguaiana/ Rio Grande do Sul, Brasil. Todos os participantes concordaram e assinaram o Termo de Consentimento Livre e Esclarecido (TCLE) para a participação no estudo. Este estudo foi aprovado pelo Comitê de Ética e Pesquisa da UNIPAMPA, sob o CAAE ํo 69695817.9.0000.5323.

Nesse sentido, prestou-se assistência a 30 tabagistas, durante o período de 60 dias, combinando atendimentos de terapia em grupo, atendimentos individuais, que incluiu 0 acompanhamento farmacoterapêutico, e a disponibilização de medicamentos. A avaliação clínica inicial foi realizada por profissional farmacêutico e os usuários foram encaminhados, conforme necessidade, a outros profissionais do programa, como médico, psicólogo, fisioterapeuta, nutricionista e enfermeiro, de forma a proporcionar um cuidado integral.

\section{Medicamentos utilizados para o tratamento do tabagismo}

A assistência prevê, conforme necessidade, a possibilidade de disponibilização de medicamentos, como dispositivos contendo Nicotina (Terapia de Reposição Nicotínica) e Cloridrato de Bupropiona, antidepressivo de primeira escolha para o tratamento do tabagismo. Estes medicamentos irão atuar na amenização dos sintomas de abstinência e na diminuição da compulsão pelo uso, aumentando a probabilidade de controle do tabagismo (BRASIL, 2001; BRASIL, 2015; BRASIL, 2016). A prescrição dos medicamentos foi de responsabilidade dos profissionais médicos e farmacêuticos, mediante avaliação clínica de cada caso.

\section{Atendimentos individuais}

A coordenação da assistência aos usuários tabagistas foi centrada nos profissionais farmacêuticos. Nesse sentido, antes de iniciar os atendimentos, cada usuário participou uma avaliação clínica, a fim de ser informado sobre o fluxograma dos atendimentos e os aspectos gerais do tratamento. Em seguida, foram programadas as consultas farmacêuticas que visaram um acompanhamento farmacoterapêutico dos pacientes de forma individual e personalizada. Além das consultas com farmacêuticos, o usuário contou com uma equipe multiprofissional (composta por médico, psicólogo, fisioterapeuta, nutricionista e enfermeiro), que realizou atendimentos individuais sempre que necessário por meio de encaminhamentos.

\section{Acompanhamento clínico dos usuários tabagistas}

De forma a avaliar e acompanhar clinicamente os indivíduos, foi realizada uma avaliação da condição geral de saúde dos usuários, que incluiu análise da farmacoterapia. O acompanhamento da evolução do tratamento foi realizado por meio da avaliação do grau de dependência à nicotina ou Teste de Fagerström, da Motivação e do o número de cigarros consumidos por dia. A avaliação da presença de sintomas relacionados à ansiedade, depressão e estresse foi verificada por meio da avaliação do grau de morbidade psicológica (Escala de Ansiedade, Depressão e Estresse- EADS-21 (LOVIBOND P e LOVIBOND S, 1995; FAGERSTRÖM KO e SCHNEIDER NG, 1989; PROCHASKA JO e DICLEMENTE CC, 1982). As respectivas avaliações foram realizadas no início e no final do período de acompanhamento de 60 dias e, após, os resultados foram analisados estatisticamente.

\section{Metodologia utilizada na terapia de grupo}

Os atendimentos de terapia em grupo ocorreram semanalmente, nas quintas-feiras, em dois turnos distintos, manhã (10:30 às 11:30 horas) e noite (18:30 às 19:30 horas). Os grupos foram integrados por 15 usuários e os encontros tiveram a duração de 60 minutos. O cronograma das reuniões do grupo foi de 8 semanas consecutivas, sendo que os usuários participantes do estudo apresentaram uma frequência igual ou superior a $70 \%$ nos encontros. A metodologia do Grupo Terapêutico de Controle do Tabagismo segue a proposta do Manual "Deixando de Fumar sem Mistérios (BRASIL, 2001), com algumas modificações pontuais de forma a incluir a Política de Redução de Danos (BRASIL, 2005). O método é estruturado a auxiliar os tabagistas a deixarem de fumar ou reduzirem o hábito, fornecendo-lhes todas as informações e estratégias que podem ser úteis nesse processo. As sessões contêm elementos significativos para auxiliar tabagistas a pararem de fumar e permanecerem sem cigarros, sendo abordados os comportamentos, pensamentos e sentimentos dos fumantes. 
A interação em grupo é utilizada para incentivar e apoiar mudanças, estruturar o senso de corresponsabilidade no tratamento e autonomia dos usuários, tornando-os aptos a compreenderem melhor seus estados de saúde e serem protagonistas de suas próprias histórias.

\section{Análise de dados}

Os dados coletados foram analisados através do programa IBM SPSS Statistics $20 \circledast$, sendo que os dados com distribuição linear foram analisados pelo Teste $T$ e os dados com distribuição não linear pelo MannWhitney, sendo consideradas diferenças estatisticamente significativas quando $p<0,05$.

\section{RESULTADOS E DISCUSSÃO}

\section{Eficácia do tratamento}

$\mathrm{Na}$ avaliação clínica inicial, os 30 tabagistas afirmaram possuir como objetivo final a interrupção do hábito, mesmo havendo a possibilidade de redução da quantidade consumida ao dia. Nesse sentido, constatou-se que a adaptação metodológica de assistência pelo PCTU, que inclui a prática de redução de danos e a política de Humanização, é uma alternativa que aproxima o usuário dos profissionais e facilita a abordagem terapêutica ao considerar os desejos do usuário no processo de cuidado, propiciando um aumento na adesão do tratamento (BRASIL, 2005; BRASIL, 2004).

$\mathrm{Na}$ avaliação clínica inicial $86,7 \%$ usuários foram considerados tabagistas pesados uma vez que consumiam uma quantidade $\geq 20$ cigarros/dia (BRASIL, 2009), segundo o teste de Fagerström, a maior parte dos tabagistas apresentou dependência elevada (50\%) ou muito elevada (23,3\%) (FAGERSTRÖM KO e SCHNEIDER NG, 1989).

Os usuários realizaram o tratamento de forma orientada pela equipe multiprofissional do PCTU e, ao final do período de 60 dias de acompanhamento terapêutico, 15 (50\%) indivíduos atingiram a meta da abstinência, $13(43,3 \%)$ reduziram, em no mínimo 50\%, a quantidade consumida ao dia e, apenas, 2 (6,6\%) não alteraram de forma significativa seu consumo, apresentando uma redução de aproximadamente $20 \%$. Conforme demonstrado no Figura 1, houve diferença estatisticamente significativa para os parâmetros de motivação, dependência nicotínica, número de cigarros e EADS-21 $(p<0,05)$, demonstrando que as intervenções planejadas contribuíram de forma estatisticamente significativa para o controle do tabagismo (redução e ou cessação) e para a diminuição dos sintomas de ansiedade, depressão e estresse.

Figura 1 - Acompanhamento clínico dos tabagistas durante o período de 60 dias.

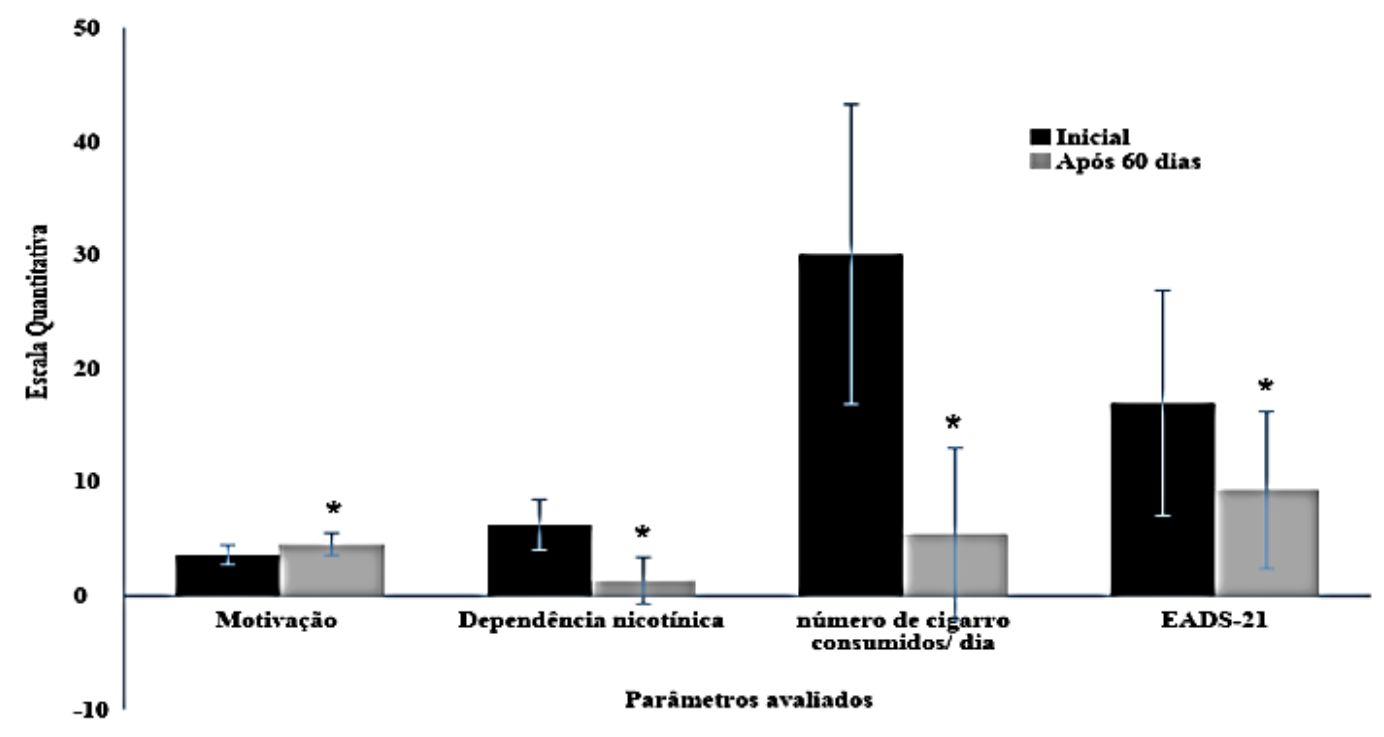

* Diferença estatisticamente significativa $(p<0,05)$. Fonte: BRUSTOLIN M, et al., 2019. 
Evidenciou-se que há uma forte influência do quadro emocional sobre o padrão de consumo de tabaco, sendo um fator bastante presente no quadro clínico desses usuários. Estudos sugerem que os tabagistas relatam maior consumo de tabaco ao sentirem-se tristes, ansiosos, bravos ou estressados devido à expectativa de alívio de sintomas negativos ou do afeto negativo (KASSEL JD, et al., 2003; COPELAND AL, et al., 1995; SPIELBERGER CD e JACOBS GA, 1982).

Entretanto, ao contrário dessa crença popular, o aparente efeito relaxante do consumo do tabaco é mascarado pela contradição da abstinência, que acaba gerando mais tensão e irritabilidade aos tabagistas (PARROTT AC, 1999; PROCHASKA JO e DICLEMENTE CC, 1982).

Os resultados encontrados estão de acordo com evidências científicas que sugerem que a interrupção do consumo de tabaco diminui os níveis de estresse, sintomas depressivos, assim como 0 afeto negativo do indivíduo, conduzindo a um aumento da qualidade de vida (PECKHAM E, MITCHELL N e BECQUE T, 2015; CASTRO MGT, et al, 2007; CHASSIN L, et al., 2002).

Ao contrário, a existência de doenças psiquiátricas ativas, como depressão, ansiedade e abuso de drogas, aumenta o risco e a probabilidade para início do tabagismo ou para o agravo da dependência nicotínica, dificultando o tratamento dessas patologias (BRESLAU N, NOVAK SP e KESSLER RC, 2004).

Todos os 30 usuários atendidos utilizaram a combinação dos medicamentos Cloridrato de Bupropiona 150 $\mathrm{mg}$ e dispositivos de Nicotina disponibilizados durante o período de tratamento. Nesse sentido, tanto no início quanto ao final do tratamento, os medicamentos foram considerados por todos usuários, a medida terapêutica mais relevante para o controle do tabagismo, havendo afirmações frequentes de que se não existissem esses dispositivos dificilmente iriam conseguir o controle do tabagismo. Este dado confirma-se à medida que 28 $(93,3 \%)$ usuários que fizeram uso de medicamento cessaram ou reduziram significativamente o hábito de fumar.

Diante do exposto, constata-se a pertinência da assistência farmacêutica aos usuários, uma vez que há, por parte dos tabagistas, uma expectativa inicial relacionada aos medicamentos, que tende a se reafirmar ao longo do processo terapêutico devido aos efeitos perceptíveis de diminuição da abstinência e da compulsão pelo hábito de fumar. Vale ressaltar que o acompanhamento farmacoterapêutico destacou-se por descentralizar o cuidado da lógica médica, conferindo à ação uma visão inovadora de evolução de práticas em saúde que favorece o usuário e o farmacêutico.

O primeiro passa a ser melhor atendido e o segundo ganha mais autonomia e reconhecimento de sua potência como profissional nos processos de cuidado em saúde. Nesse sentido, o vínculo entre os usuários e farmacêuticos proporcionou aderência e efetividade ao tratamento ao possibilitar a correta orientação da farmacoterapia, manejo de problemas relacionados ao aparecimento de reações adversas, bem como a resolução de dúvidas e o repasse de orientações e esclarecimentos de todo o processo de cuidado, o que conferiu uma visão inovadora e diferenciada a toda a assistência prestada pelo Programa para Controle do Tabagismo (PCT).

Para os casos em que a bupropiona e a nicotina não apresentaram uma contribuição efetiva no tratamento, há outras opções farmacológicas como a vareniclina, clonidina e nortriptilina (BRASIL, 2009). Além disso, suspeita-se que nesses casos o tabagismo esteja mais associado a fatores psicológicos e comportamentais do que propriamente à abstinência da nicotina, conferindo uma análise mais complexa relacionada à dependência do tabaco.

Estudos afirmam que a dependência nicotínica está relacionada à genética e, dessa forma, codificações específicas nos receptores nicotínicos contribuiriam para um aumento no risco de desenvolvimento da dependência entre fumantes (BIERUT LJ, JOHNSON EO e SACCONE NL, 2014), fato que também poderia explicar a ineficácia do tratamento nesses usuários. Nesses casos, as abordagens de terapia cognitivocomportamental seriam medidas terapêuticas promissoras, mas que provavelmente dispensariam de um período de tempo maior que a terapia farmacológica para que os resultados fossem observados (POWELL VB et al, 2008). 
Ao final do acompanhamento terapêutico pode-se evidenciar que a análise da motivação juntamente com o teste de Fagerström foram bons preditores para prognóstico do processo de controle do tabagismo, entretanto, verifica-se que há mecanismos psicocomportamentais subjetivos envolvidos no processo de dependência que estão relacionados ao nível de conscientização, à determinação empregada no processo, à capacidade de recepção, compreensão e internalização de novos pensamentos, existência de sintomas relacionados à morbidade psicológica e ao ambiente social que o indivíduo habita. Nesse sentido, intervenções que envolvam uma escuta ativa do paciente, que gerem reflexões e a consequente compreensão dos motivos que o levam a fumar, são extremamente importantes para o estabelecimento de estratégias para o controle do tabagismo.

\section{Acompanhamento farmacoterapêutico}

O acompanhamento por profissional farmacêutico possibilitou a orientação do uso racional dos medicamentos e dos cuidados complementares, utilizando, conforme necessidade, a prescrição farmacêutica para a indicação de utilização de medicamentos (normalmente dispositivos de reposição de nicotina) ou mesmo de cuidados complementares de saúde, como a prática de atividades físicas, dicas a respeito da alimentação, dentre outros. Além disso, o acompanhamento incluiu um processo de avaliação clínica contínua, possibilitando uma análise a respeito do padrão psicocomportamental de consumo do tabaco, que se constituiu um importante instrumento para a formulação de estratégias terapêuticas individuais de controle do tabagismo.

Os profissionais farmacêuticos na condução dos processos de cuidado e na inovação da assistência em saúde, considerando que o acompanhamento clínico individual e contínuo desses usuários, possibilita uma análise integral do padrão comportamental de consumo do tabaco desses indivíduos, constituindo um importante instrumento para a formulação de estratégias terapêuticas individuais de controle do tabagismo, conferindo maior efetividade e segurança ao tratamento. Sendo assim é possível afirmar que a Assistência Farmacêutica tem apotencial de dar apoio e ser parte integrante do conjunto de ações e de serviços do Sistema Único de Saúde (BRASIL, 2014).

\section{A Terapia de Grupo}

A terapia de grupo complementou a assistência, esclarecendo pontos importantes a respeito da dependência e contribuiu para a conscientização a respeito do processo de controle do tabagismo. Ainda, verificou-se que a coletividade estimula e fortalece a motivação dos usuários no processo, uma vez que há a percepção e análise de diferentes pontos de vista que podem se complementar e favorecer uma ação mais integral do cuidado, aumentando as probabilidades do usuário alcançar seus objetivos.

A terapia cognitivo-comportamental, técnica empregada tanto em atendimentos individuais como em grupo, envolve o estímulo ao autocontrole ou automanejo para que o indivíduo possa aprender como escapar do ciclo vicioso da dependência, tornando-se um agente de mudança de seu próprio comportamento (BRASIL, 2001; BRASIL, 2016). Segundo, Yalom ID (2006), a terapia de grupo é uma forma de psicoterapia bastante efetiva, não menos que a individual, tratando-se de um processo bastante complexo que ocorre a partir da interação de diferentes experiências. Essa ação disponibiliza um princípio organizacional que possibilita a seleção de estratégias de forma a maximizar a potência da experiência para o alcance dos objetivos do grupo.

\section{CONCLUSÃO}

A assistência ofertada pelo PCTU aos tabagistas demonstrou ser efetiva em 60 dias de acompanhamento, auxiliando a maior parte dos usuários a alcançarem seus objetivos. Assim, o controle do tabagismo manifestado pela cessação ou redução do consumo do tabaco resultou em uma diminuição significativa da morbidade psicológica e contribuiu, consequentemente, para uma diminuição dos riscos relacionados ao aparecimento de comorbidades físicas e mentais. Neste estudo foram identificados como limitações o número reduzido de indivíduos participantes do grupo, período de acompanhamento e baixo número de estudos publicados na língua portuguesa. 


\section{REFERÊNCIAS}

1. AMERICAN PSYCHIATRIC ASSOCIATION. Manual diagnóstico e estatístico de transtornos mentais [recurso eletrônico]: DSM-5 / [American Psychiatric Association; tradução: Maria Inês Corrêa Nascimento. et al.]; revisão técnica: Aristides Volpato Cordioli.[et al.]. - 5. ed. - Dados eletrônicos. - Porto Alegre: Artmed, 2014.

2. BRASIL. Primeiras diretrizes clínicas na saúde suplementar - versão preliminar / organizado por Agencia Nacional de Saúde Suplementar, Associação Medica Brasileira. - Rio de Janeiro: ANS, 2009.

3. BRASIL. Ministério da Saúde. Instituto Nacional de Câncer - INCA. Coordenação de Prevenção e Vigilância (CONPREV). Abordagem e Tratamento do Fumante - Consenso 2001. Rio de Janeiro: INCA, 2001.

4. BRASIL. Ministério da Saúde. Secretaria de Atenção à Saúde. Departamento de Atenção Básica. Estratégias para o cuidado da pessoa com doença crônica: o cuidado da pessoa tabagista / Ministério da Saúde, Secretaria de Atenção à Saúde, Departamento de Atenção Básica. - Brasília: Ministério da Saúde, 2015. 154 p.: il. (Cadernos da Atenção Básica, n. 40) ISBN 978-85-334-2339-8

5. BRASIL. Ministério da Saúde. Secretaria de Atenção à Saúde. Portaria no 761, de 21 de junho de 2016. Valida as orientações técnicas do tratamento do tabagismo constantes no Protocolo Clínico e Diretrizes Terapêuticas Dependência à Nicotina.

6. BRASIL. Ministério da Saúde. Secretaria de Atenção à Saúde. Instituto Nacional do Câncer. Coordenação de Prevenção e Vigilância. Deixando de Fumar sem Mistérios- Rio de Janeiro: INCA, 2008.

7. BRASIL. Ministério da Saúde. Portaria ํㅜ 1.028, de 1ำ de Julho de 2005. Determina que as ações que visam à redução de danos sociais e à saúde, decorrentes do uso de produtos, substâncias ou drogas que causem dependência, sejam reguladas por esta portaria. 2005.

8. BRASIL. Ministério da Saúde. Núcleo Técnico da Política Nacional de Humanização. HumanizaSUS: política nacional de humanização: documento base para gestores e trabalhadores do SUS. 2. Ed. - Brasília: Ministério da Saúde, 2004.

9. BRESLAU N, NOVAK SP, KESSLER RC. Psychiatric Disorders and stages of Smoking. Biological Psychiatry 2004; 55:69-76.

10. BIERUT LJ, JOHNSON EO, SACCONE NL. A glimpse into the future - Personalized medicine for smoking cessation. Neuropharmacology. 2014; 76.

11. CASTRO MGT, et al. Qualidade de vida e gravidade da dependência de tabaco. Revista de psiquiatria clínica, 2007; 34(2): 61-67.

12. CHASSIN L, PRESSON C, SHERMAN S, KIMK. Long-Term Psychological sequelae of Smoking Cessation and Relapse. American Psychological Association, 2002; 21(5):438-443.

13. CHASSIN L, et al. Long-Term Psychological sequelae of Smoking Cessation and Relapse. American Psychological Association, 2002; 21 (5): 438-443.

14. COPELAND AL, et al. The Smoking Consequences Questionnaire-Adult: Measurement of smoking outcome expectancies of experienced smokers. Psychological Assessment, 1995; 7(4), 484-494.

15. FAGERSTRÖM KO, SCHNEIDER NG. Measuring nicotine dependence: a review of the Fagerström Tolerance Questionnaire. Journal of Behavioral Medicine, [S.I.], 1989; 12(2):159-182.

16. INCA- Instituto Nacional do Câncer. Ministério da Saúde. Programa Nacional de Controle do Tabagismo. Rio de Janeiro, 2017.

17. KASSEL JD, et al. Smoking, stress, and negative affect: Correlation, causation, and context across stages of smoking. Psychological Bulletin, 2003; 129(2): 270-304.

18. MOREIRA EDT. Classificação de Cigarros Usando Espectrometria NIRR e Métodos Quimiométricos de Análise. Dissertação (Mestrado em Química) - Universidade Federal da Paraíba. João Pessoa, PB, 2007.

19. OLIVEIRA F, COSTA MCF. Cultivo de Fumo (Nicotianatabacum L.). Dossiê Técnico- Universidade de São Paulo USP, 2012.

20. PICCIOTTO MR, MINEUR YS. Molecules and circuits involved in nicotine addiction: The many faces of smoking. Neuropharmacology, 2014; 76:545-553.

21. RIAD-ALLEN L, DERMODY SS, HERMAN Y, BELLISSIMO K, SELBY P, GEORGE TP et al. Becoming TobaccoFree: Changes in Staff and Patient Attitudes and Incident Reports in a Large Academic Mental Health and Addictions Hospital. The American Journal on Addictions, XX: 1-9, 2017. American Academy of Addiction Psychiatry.

22. ROSEMBERG J. Nicotina: droga universal. Monografia. Produção Independente. São Paulo: 2004.

23. PARROTT AC. Does cigarette smoking cause stress? American Psychologist, 1999; 54(10):817-820.

24. PECKHAM E, MAN MS, MITCHELL N, LI J, BECQUE T, KNOWLES S, et al. Smoking Cessation Intervention for severe Mental III Health Trial (SCIMITAR): a pilot randomised control trial of the clinical effectiveness and costeffectiveness of a bespoke smoking cessation service. Health Technol Assess 2015; 19(25).

25. PROCHASKA JO, DICLEMENTE CC. Transtheoretical therapy toward a more integrative model of change. Psychother Theory Res Pract. 1982:19(3):276-87.

26. POWEL, VB, ABREU N, OLIVEIRA, IE et al. Cognitive-behavioral therapy for depression. Rev Bras Psiquiatr. 2008; 30 (Supl II):S73-80

27. LOVIBOND P, LOVIBOND S. The structure of negative emotional states: Comparison of the depression anxiety stress scales (DASS) with the Beck Depression and Anxiety Inventories. Behavior Research and Therapy, New Brunswick, 1995; 33(3):335-343.

28. SPIELBERGER CD, JACOBS GA. Personality and smoking behavior. Journal of Personality Assessment, 1982;46:396-403. 
29. YALOM ID. Psicoterapia de Grupo - Teoria e prática. Porto Alegre: Artmed, 2006, 23-36.

30. VIGITEL- Vigilância de Fatores de Risco e Proteção para Doenças Crônicas por Inquérito Telefônico. Estimativas sobre frequência e distribuição sócio-demográfica de fatores de risco e proteção para doenças crônicas nas capitais dos 26 estados brasileiros e no Distrito Federal. 2015.

31. WHO- World Health Organization. Policy Recommendations for Smoking Cessation and Treatment of Tobacco Dependence. Geneve, WHO, 2003.

32. WHO- World Health Organization. Tobacco and its environmental impact: an overview. Geneva: World Health Organization 2017. 